\title{
Does preoperative dipyridamole-thallium scanning reduce 90-day cardiac complications and mortality in patients with femoral neck fractures undergoing hemiarthroplasty?
}

\section{Chin-Yi Liao}

Chang Gung Memorial Hospital Kaohsiung Branch

Timothy L. Tan

Rothman Orthopaedic Institute

Yu-Der Lu

Chang Gung Memorial Hospital Kaohsiung Branch

Cheng-Ta Wu

Chang Gung Memorial Hospital Kaohsiung Branch

Mel S Lee

Chang Gung Memorial Hospital Kaohsiung Branch

Feng-Chih Kuo ( $\nabla$ fongchikuo@cgmh.org.tw)

Chang Gung Memorial Hospital Kaohsiung Branch https://orcid.org/0000-0001-5205-8333

Research article

Keywords: dipyridamole-thallium scan, femoral neck fracture, mortality, cardiac complications, hemiarthroplasty.

Posted Date: March 4th, 2020

DOl: https://doi.org/10.21203/rs.3.rs-15965/v1

License: (c) (1) This work is licensed under a Creative Commons Attribution 4.0 International License. Read Full License 


\section{Abstract}

Background: This study aimed to evaluate the effect of dipyridamole-thallium scanning on 90-day cardiac complications and mortality in patients with femoral neck fracture treated with hemiarthroplasty. Methods : Between 2008 and 2015, 844 consecutive patients undergoing cemented or cementless hemiarthroplasty were identified from the database of a single level-one medical center. One-hundred and thirteen patients (13.4\%) underwent the dipyridamole-thallium scan test prior to surgery, and 731 patients $(86.6 \%)$ did not. Patient characteristics, comorbidities, surgical variables, and duration to surgery were recorded. Multivariate logistic regression was performed, and a propensity-score-matched cohort was identified in order to determine whether dipyridamole-thallium scanning was associated with 90-day cardiac complications and mortality. Results: The incidence of 90 -day cardiac complications was $19.47 \%$ in the thallium scan group and $11.58 \%$ in the non-thallium scan group $(p=0.343)$ among 452 patients after propensity score-matching. The 90 -day mortality rate was similar in the two groups $(0.88 \% \mathrm{vs}$. $1.18 \%, p=0.795)$. In the propensity score-matched patients, utilization of the dipyridamole-thallium scan was not associated with cardiac complications (matched cohort, adjusted odds ratio [OR] $=1.32 ; 95 \%$ confidence interval [Cl] 0.75 to 2.33 ) or mortality (adjusted $\mathrm{OR}=0.75 ; 95 \% \mathrm{Cl} 0.08$ to 6.71 ) within 90 days. Risk factors for cardiac complications were an American Society of Anesthesiologists grade 3 (OR 3.19, $95 \% \mathrm{Cl} 1.44$ to 7.08 ) and pre-existing cardiac comorbidities (OR 5.56, 95\% $\mathrm{Cl} 3.35$ to 9.25). Discussion : Preoperative dipyridamole-thallium scanning was not associated with reductions in 90-day cardiac complications and mortality in patients with femoral neck fracture undergoing hemiarthroplasty. The necessity for dipyridamole-thallium scanning should be re-evaluated in elderly patients with femoral neck fractures, given that this increases the duration to surgery.

\section{Introduction}

Femoral neck fracture is a very common geriatric low-energy trauma that has profound economic and medical impacts worldwide [1, 2]. The standard treatment for displaced femoral neck fractures in geriatric patients is often hemiarthroplasty [3]. However, patients may experience significant morbidity and mortality due to pre-existing cardiovascular comorbidities and insult from surgery [4]. Therefore, it is common practice that there is a preoperative consultation with internal medicine doctors or anesthesiologists for risk stratification and treatment optimization.

Dipyridamole-thallium scanning has been suggested as a method of preoperatively assessing cardiac risk in patients undergoing major non-cardiac surgery. The American College of Cardiology (ACC) and American Heart Association (AHA) guidelines also adopt dipyridamole-based thallium scanning as one of the modalities for perioperative cardiovascular evaluation prior to noncardiac surgery $[5,6]$. In terms of preoperative practice in other specialties, for instance, Landesberg et al. [7] described the characteristics of thallium scanning and selective coronary angiography prior to major vascular therapy and reported a significant improvement in the long-term outcome. Yin et al. [8] also demonstrated that applying preoperative dipyridamole thallium-201 scintigraphy can prevent cardiac mortality and complications in patients undergoing parathyroidectomy. Hemiarthroplasty is a non-cardiac procedure that is classified as 
posing an intermediate cardiac risk in $1-5 \%$ of patients in the ACC/AHA preoperative guidelines [5]. However, there is minimal literature on whether this exam results in any differences in outcomes in patients undergoing hip fracture surgery.

The purpose of this study was to evaluate the correlations of dipyridamole-thallium scanning with 90-day cardiac complications and mortality in patients with femoral neck fracture treated with hemiarthroplasty.

\section{Material And Methods}

After institutional review board approval, we retrospectively enrolled 968 consecutive patients undergoing hemiarthroplasty from a trauma database at a single academic institution from January 2008 to December 2015. The inclusion criteria were patients with displaced femoral neck fractures who were older than 60 years. Patients with a non-displaced fracture, those aged under 60 years, and those with multiple trauma, valgus impacted fracture, or open fracture were excluded. After the application of the exclusion criteria, 844 patients with displaced femoral neck fractures undergoing cemented or cementless hemiarthroplasty were included in this study.

All patients underwent a preoperative medical examination that included a resting electrocardiogram, chest radiography and blood exam. Following this screening, they were seen by an anesthesiologist before surgery. Subsequently, a cardiology consultation was obtained if concerns were raised by the anesthesiologist. The cardiologist or physician evaluated the patients undergoing non-cardiac surgery based on the 2007 ACC/AHA guidelines on perioperative cardiovascular evaluation [5]. As per the guidelines, routine screening with non-invasive stress testing was not performed in patients undergoing low-risk non-cardiac surgery. However, if the test result may have affected our decision-making, including canceling the operation or changing the perioperative care, it was arranged for patients with an elevated cardiac risk and a poor metabolic equivalent of task (MET < 4). If the patient had an elevated cardiac risk but a moderate functional capacity (MET >4), no further testing was required.

Patients were divided into two categories based on receipt of a dipyridamole-based thallium scan. The control group included patients who underwent surgery without a thallium scan after consultation with an anesthesiologist or cardiologist, while the study group included patients who received a dipyridamolethallium scan. Patients with abnormal thallium scan results were further evaluated by a cardiologist with regards to coronary angiography intervention. Indications for coronary angiography were based on clinical findings, such as new or medically unstable angina, previous or recent myocardial infarction, or persistent angina. Stent implantation or balloon angioplasty during angiography was performed if coronary arterial stenosis was greater than $50 \%$, the multiple-vessel disease was present, or if left main coronary artery occlusion was observed.

Variables of interest were extracted by electronic query of medical records and were reviewed manually. The following information was obtained: basic demographic data, age, gender, body mass index (BMI), ASA grade, and preoperative comorbidities, including ischemic heart disease, congestive heart failure, valvular heart disease, cerebrovascular accident (CVA), diabetes, chronic obstructive pulmonary disease 
(COPD), rheumatoid arthritis, liver disease, renal disease, and a history of cancer. In addition, the duration from hospital presentation to surgery, anesthesia type (regional or general), use of a cemented or cementless stem, operation duration, and need for transfusion were also obtained.

All displaced femoral neck fractures were treated with hemiarthroplasty using a posterolateral approach in a lateral decubitus position. All patients were treated using a cemented or cementless fixation technique by well-trained orthopedic surgeons. The cemented or press-fit implants were chosen based on preoperative imaging evaluation for osteoporosis and intraoperative trial implantation at the discretion of the treating surgeons. Intra-operative joint capsules were approximated by sutures, and a closed drainage tube was inserted, which was removed within 24-48 hours. A partial weight-bearing rehabilitation program was initiated by a physical therapist from postoperative day 1 . If a patient was unable to ambulate using crutches, wheelchair ambulation was recommended.

\section{Outcome measurement}

The outcomes of this study included cardiac complications and mortality within 90 days after the index hemiarthroplasty. The cardiac complication was a composite of coronary disease, heart failure, arrhythmia, or acute myocardial infarction, while mortality was defined as any death after the surgery. We traced all medical records after discharge and made phone calls if patients were lost to follow-up through outpatient clinics for post-op 90 days.

\section{Statistical analysis}

All variables were tested for normality using the Kolmogorov-Smirnov test. Student's t-test was utilized for normally-distributed data, and the Mann-Whitney U test was used for nonparametric data. Fisher's exact test or the chi-squared test was used for categorical variables. We used the propensity score-matching (at a 1:3 ratio) technique with a logistic regression model to adjust the covariates, including basic demographic data (age, gender, BMI and ASA grade), preoperative comorbidities (all cardiac comorbidities, CVA, diabetes, COPD, rheumatoid disease, liver disease, renal disease, and cancer history), surgical variables (anesthesia type, use of a cemented or cementless stem, operation duration, and transfusion) and duration to surgery, in order to decrease the differences in baseline conditions between groups and to minimize the selection bias of thallium scan application. Univariate and multivariate logistic regression analysis was also applied to determine whether or not dipyridamole-thallium scanning was an independent factor affecting the 90-day cardiac complications and mortality after adjusting for the confounding factors listed above. Odds ratios (ORs) with 95\% confidence intervals (Cls) were calculated, and a p-value of less than 0.05 was used to evaluate statistical significance. All statistical analyses were performed using the Statistical Package for Social Science (SPSS) version 22 software (SPSS Inc., IBM, Armonk, New York, USA) and the NCSS Statistical Analysis and Graphics software program (NCSS, LLC, Kaysville, Utah, USA).

\section{Results}


There were several baseline differences between those who received a preoperative dipyridamole-thallium scan and those who did not before propensity score-matching. Patients who underwent a dipyridamolethallium scan were of an older age $(p<0.001)$ and a higher ASA grade $(p<0.001)$, with a higher proportion of patients with ischemic heart disease $(22.1 \%$ vs $7.7 \%, p=0.001)$, valvular heart disease ( $9.7 \%$ vs $2.5 \%, p=0.001$ ), and diabetes mellitus ( $38.9 \%$ vs $29.5 \%, p=0.044)$, and cemented hemiarthroplasty was more common in these patients ( $57.5 \%$ vs. $40.6 \%, p<0.001)$. Furthermore, patients who underwent a dipyridamole-thallium scan had to wait an average of 4.6 days longer to receive surgery (7.0 \pm 12.4 days vs. $2.4 \pm 3.3$ days, $p<0.001)$.

After performing propensity score-matching, patients who underwent a dipyridamole-thallium scan had a higher ASA grade $(p=0.01)$, and a higher proportion of those patients had ischemic heart disease $(p=$ 0.038). Further standardized difference analysis of ASA grade showed a reduction from $62.8 \%$ to $32.1 \%$, and the standardized difference of ischemic heart disease was reduced from $36.0 \%$ to $18.0 \%$. All other covariates and comorbidities were statistically balanced after each examination of the Student $t$-test and chi-square test results. Patients who underwent a thallium scan still had a longer preoperative duration before surgery $(7.0 \pm 12.4$ days vs. $3.7 \pm 4.2$ days, $p<0.001)$.

In our study, 90-day cardiac complications included 2 patients with angina pectoralis, 32 with symptomatic heart failure, 60 with cardiac arrhythmia, 1 with thoracic aortic dissection and 6 with acute myocardial infarction. After propensity score matching, the incidence of 90-day cardiac complications in a matched cohort of 452 was $19.47 \%$ in the dipyridamole-thallium scan group and $15.58 \%$ in the nondipyridamole-thallium scan group $(p=0.343)$. Furthermore, the 90 -day mortality was similar between the two groups $(0.88 \%$ vs. $1.18 \%, p=0.795)$ (Fig. 1$)$.

The correlations of dipyridamole-thallium scan with 90-day cardiac complications and mortality are depicted in Table 2. Before propensity score-matching, univariate logistic regression revealed a significant difference in terms of the correlation between thallium scanning and postoperative cardiac complications ( $p=0.019$, OR $1.86,95 \% \mathrm{Cl} 1.11$ to 3.13 ), but no statistically significant difference was observed for 90 day mortality $(p=0.941)$. When controlling for potential confounders, multivariate analysis revealed no significant association between dipyridamole-thallium scanning and 90-day cardiac complications (adjusted OR $=1.44 ; 95 \% \mathrm{Cl} 0.71$ to $2.91, p=0.309$ ) or 90-day mortality (adjusted OR $=9.80 ; 95 \% \mathrm{Cl} 0.23$ to $424.80, p=0.235$ ). Analysis of the propensity score-matched cohort also confirmed no significant differences in terms of the correlation between dipyridamole-thallium scanning and 90-day cardiac complications (adjusted OR $=1.32 ; 95 \% \mathrm{Cl} 0.75$ to $2.33, p=0.332$ ) or 90 -day mortality (adjusted OR= $0.75 ; 95 \% \mathrm{Cl} 0.08$ to $6.71, p=0.797$ ). We performed further subgroup evaluation of cardiac complications in order to assess the correlations of thallium scan with postoperative unstable angina and acute myocardial infarction, and no statistically significant differences between the thallium scan group and the non-thallium scan group were observed ( $p=0.437$ and 0.272 , respectively).

Independent risk factors for post-operative 90-day cardiac complications included an ASA grade 3 (OR $3.19,95 \% \mathrm{Cl} 1.44$ to $7.08, p=0.004)$ and pre-existing cardiac comorbidity (OR 5.56, 95\% $\mathrm{Cl} 3.35$ to $9.25, p$ 
$<0.001$ ) in the multivariate analysis (Table 3 ).

In the 113 patients who underwent a dipyridamole-thallium scan, 23 (20.3\%) had positive findings of reversible myocardial ischemia and 90 patients $(79.7 \%)$ had negative findings. We compared the patients with negative results and those with positive results, and analysis of the demographic data showed that higher proportions of the patients who underwent a thallium scan were male $(p=0.001)$ and had ischemic heart disease $(p<0.001)$, with statistical significance. Although there was initially a significant correlation between thallium scanning and postoperative cardiac episodes $(p=0.042)$, further logistic regression revealed no statistically significant difference (OR 6.70; $95 \% \mathrm{Cl} 0.85$ to $52.68, p=0.071)$. Of the 23 patients who were found to have reversible myocardial ischemia, 10 received cardiac catheterization, but only 3 received primary coronary interventions with stenting, either bare metal or drug-eluting stents,

and only 1 patient received balloon angioplasty. The 7 patients who did not undergo $\mathrm{PCl}$ all ultimately had surgery. None of these 10 patients had postoperative cardiac complications or mortality within 90 days.

Of the 13 patients who underwent a dipyridamole-thallium scan who did not receive cardiac catheterization, one developed postoperative myocardial infarction, but soon recovered after primary coronary intervention with stenting. We followed-up this patient who developed postoperative myocardial infarction for one postoperative year, and no further cardiac morbidity or mortality was documented (Fig. 2).

All of the 23 patients in the thallium scan group with reversible myocardial ischemia were alive at postoperative 90 days. Three patients who did not receive primary coronary interventions were dead within one year after surgery. One of those patients had probable reversible myocardial ischemia, and the leading cause of death was respiratory failure after benzodiazepine overdose. The second patient had irreversible myocardial ischemia, and the leading cause of death was heart failure with pulmonary edema. The final patient had an equivocal result of the thallium test, and the leading cause of death was pneumonia. None of the mortality was related to myocardial ischemia according to the clinical evidence.

\section{Discussion}

Preoperative medical optimization prior to surgery for hip fractures frequently requires balancing the benefits of potential medical interventions with complications arising from prolonged immobilization. Several previous studies have found that a prolonged duration to surgery ( $\geq 48$ hours) for hip fractures may increase the 30 -day and 1-year mortality rates $[9,10]$. However, patients suffering from hip fractures usually are elderly, fragile, and have many pre-existing medical conditions. Thus, it is a frequent practice that preoperative consultation with another service is obtained for risk stratification and medical optimization. While the goal is to make a decision to proceed or medically optimize the patient first, the process of preoperative medical evaluation usually takes 1 to 2 days before heading to surgery [11]. Among the common preoperative examinations, the dipyridamole-based thallium scan may require a longer duration to organize, which is often dependent on scanner availability and time of day [12-15], and a 4-day average delay was seen at our institution. 
If a thallium scan would affect the risk of postoperative complications, and if further selective preoperative treatment such as coronary angiography may be required, postoperative cardiac complications may be reduced by balancing the risks in the two groups. Thus, we utilized the propensity score-matching technique to balance the differences in baseline conditions and comorbidities between the groups and to minimize the selection bias caused by retrospective cohort study. The results of our study suggested that the rate of 90-day cardiac complications was still high despite the use of a dipyridamole-thallium scan (19.47\% vs. $15.58 \%$, Fig. 1). Utilization of preoperative dipyridamole-thallium scanning was not significantly associated with a change in the 90-day overall cardiac complications (OR $=1.32 ; 95 \% \mathrm{Cl} 0.75$ to $2.33 ; p=0.332$, Table 2 ) or mortality (adjusted OR $=0.75 ; 95 \% \mathrm{Cl} 0.08$ to $6.71 ; p=$ 0.797) after correcting the confounding factors in the multivariate analysis. Further subgroup evaluation of patients with postoperative unstable angina and acute myocardial infarction was performed, which also showed no statistically significant differences between the two groups $(p=0.437$ and 0.272 , respectively). We found that patients with ASA grade $\geq 3$ and pre-existing cardiac comorbidities (ischemic heart disease, congestive heart failure, and valvular heart disease) were associated with an increased risk of 90-day cardiac complications. Furthermore, among the patients who underwent a thallium scan, only one (4.3\%) had myocardial infarction after surgery, and no mortality was observed within the postoperative 90 days. Our results suggested that the performance of a thallium scan was not predictive of cardiac complications, despite delaying surgery for an average of 4 days.

Ricci et al. [16] demonstrated that preoperative cardiac testing (dipyridamole-thallium scanning, echocardiography, and cardiac catheterization) did not change the management of perioperative orthopedic surgery or medical therapy in elderly patients with hip fractures, but did incur a huge cost of over $\$ 47$ million annually in the United States, with a delay to surgery of on average 3.3 days, as compared with 1.9 days. Multiple studies have also demonstrated that preoperative cardiac testing with echocardiography delays surgery without a significant change in preoperative cardiac medications or anesthesia [11, 17-19]. In addition, the cost of a thallium test is 261.22 dollars at our institution. Compared with those studies, we included more patients in our study. Moreover, we performed a propensity score analysis to control possible group differences in patients' background conditions and minimize the effect of selection bias. We also analyzed the independent factors related to postoperative cardiac complications.

It is rational to consider that perioperative cardiac assessment could lead to safer surgery and fewer cardiac complications, as has been demonstrated in other specialties, such as thyroid and cardiovascular surgery $[7,8]$. However, the results of previous studies did not indicate increased benefit in cases of geriatric hip trauma. Thus, we suppose that over-screening may happen in daily practice. Currently, during preoperative assessment by a cardiologist who follows the ACC/AHA guidelines, patients who have a functional capacity of < 4 METs require further evaluation, which is arbitrary and subjective, and may lead to over-diagnosis and incorrect indications of cardiac examinations. Vigoda et al. [20] performed a study of 548 anesthesiologist residents nationwide and examined their judgement in different scenarios. Fewer than half of the participants adhered to correct practice in accordance with standard care as set down in the 2007 ACC/AHA guidelines. We also assumed that the evolving minimally-invasive technique of 
hemiarthroplasty with a reduced surgical duration, in conjunction with postoperative early rehabilitation with mechanical/pharmaceutics thromboembolism prophylaxis, would result in a lower incidence of postoperative morbidity as compared with the same surgery decades ago. There may exist an imbalance between the expensive nuclear image test, which delays surgery for up to 4 days, and its clinical benefit.

A precisely selective coronary angiography and intervention are no doubt helpful to decrease postoperative morbidity/mortality. In our study, 113 patients underwent a thallium scan, but only 10 received coronary angiography and only 3 of the 10 patients who underwent angiography required interventions with a stenting procedure (2.65\%). Thus, we identified a low rate of selective angiography with $\mathrm{PCl}$ after obtaining the results of a thallium scan, which may account for a decreased clinical benefit of testing. The thallium scan has a questionable negative predictive value in patients with "balanced ischemia", which was recognized by a condition of global myocardial ischemia. Several researchers discovered poor interpretation of a relative perfusion defect in affected segments, which is a common phenomenon in nuclear imaging [21-23].

There were several limitations of this study that should be considered. Most notably, there may be a selection bias, as dipyridamole-thallium scanning was ordered in high-risk patients who were likely inherently subject to a higher rate of 90-day cardiac complications. However, we attempted to balance the baseline patient condition and control for potential confounders. Second, the decision to utilize dipyridamole-thallium scanning was made by different cardiologists, and thus there may be some variation in the stratification and willingness to order additional cardiac tests. Third, we focused on 90day morbidity and mortality, and thus did not account for any complications that may have occurred beyond that follow-up duration. It is necessary to discover a better cardiac assessment tool with better specificity that is less time-consuming and has a better negative predictive value. Currently, there is no consensus with regards to a thallium scan substitute but computed tomographic angiography (coronary CTA) has been noted to be useful. Huang et al. [24] investigated the value of coronary CTA in non-cardiac surgeries and found that this yielded improved perioperative risk stratification with a low rate of major cardiac events, high specificity, and good negative predictive value. The risk of a major cardiac event was $14 \%$ in patients with significant CTA findings [24]. Future research involving prospective, randomized allocation of patients is warranted to minimize selection bias and group differences.

\section{Conclusion}

Preoperative dipyridamole-thallium scanning was not associated with reductions in 90-day cardiac complications and mortality in patients with femoral neck fracture undergoing cemented or cementless hemiarthroplasty. Patients who underwent a dipyridamole-thallium scan received surgery four days later on average than patients who did not, with no significant clinical benefit in terms of 90-day cardiac complications and mortality. Given the results of this study and those reported in pre-existing literature, we propose that routine dipyridamole-thallium scanning in patients with an isolated femoral neck fracture should not be performed. We thus suggest that it is important to consider the patient's preoperative cardiovascular comorbidity, underlying disease, and daily function during the preoperative assessment. 


\section{Abbreviations}

ASA, American Society of Anesthesiologists; CHF, congestive heart failure; CVA, cerebrovascular accident; COPD, chronic obstructive pulmonary disease; RA, rheumatoid arthritis; SD, standard deviation. OR, odds ratio; $\mathrm{Cl}$, confidence interval

\section{Declarations}

\section{Ethics approval and consent to participate}

The protocols used in this retrospective study was reviewed and approved by the Institutional Review Board of Chang Gung Medical Foundation (No. 201600768B0). According to the Taiwanese national legislation, patient consent is not required in retrospective studies.

\section{Consent for publication}

Not applicable.

\section{Availability of data and materials}

Data is available from the corresponding author.

\section{Competing interests}

All the authors declared that they have no competing interests.

\section{Funding}

None.

\section{Authors' contributions}

CY Liao and FC Kuo were involved in the conception and design of the study. CY Liao, FC Kuo and YD Lu and CT Wu collected and analyzed the data together. CY Liao and TL Tan were involved in literature search and manuscript drafting. MSL and FC Kuo supervised the version to be published. All authors read and approved the final manuscript.

\section{Acknowledgment}

We appreciated the Biostatistics Center, Kaohsiung Chang Gung Memorial Hospital for work.

\section{Author details}

${ }^{1}$ Department of Orthopaedic Surgery, Kaohsiung Chang Gung Memorial Hospital, College of Medicine, Chang Gung University, Kaohsiung, Taiwan 
${ }^{2}$ Rothman Orthopaedic Institute at Thomas Jefferson University, Philadelphia, Pennsylvania, United States

\section{References}

1. Wu TY, Hu HY, Lin SY, Chie WC, Yang RS, Liaw CK. Trends in hip fracture rates in Taiwan: a nationwide study from 1996 to 2010. Osteoporos Int. 2017;28:653-65. doi:10.1007/s00198-0163783-4.

2. Kanis JA, Odén A, McCloskey EV, Johansson H, Wahl DA, Cooper C, et al. A systematic review of hip fracture incidence and probability of fracture worldwide. Osteoporos Int. 2012;23:2239-56. doi:10.1007/s00198-012-1964-3.

3. Zielinski SM, Meeuwis MA, Heetveld MJ, Verhofstad MHJ, Roukema GR, Patka P, et al. Adherence to a femoral neck fracture treatment guideline. Int Orthop. 2013;37:1327. doi:10.1007/s00264-013-18883.

4. D’Angelo F, Giudici M, Molina M, Margaria G. Mortality rate after hip hemiarthroplasty: analysis of risk factors in 299 consecutives cases. J Orthop Traumatol. 2005;6:111-6. doi:10.1007/s10195-0050093-6.

5. Fleisher LA, Beckman JA, Brown KA, Calkins H, Chaikof EL, Fleischmann KE, et al. ACC/AHA 2007 Guidelines on Perioperative Cardiovascular Evaluation and Care for Noncardiac Surgery: A Report of the American College of Cardiology/American Heart Association Task Force on Practice Guidelines (Writing Committee to Revise the 2002 Guideline. Circulation. 2007;116:e418-500. doi:10.1161/CIRCULATIONAHA.107.185699.

6. Fleisher LA, Fleischmann KE, Auerbach AD, Barnason SA, Beckman JA, Bozkurt B, et al. 2014 ACC/AHA guideline on perioperative cardiovascular evaluation and management of patients undergoing noncardiac surgery: a report of the American College of Cardiology/American Heart Association Task Force on Practice Guidelines. Circulation. 2014;130:e278-333. doi:10.1161/CIR.0000000000000106.

7. Landesberg G, Mosseri M, Wolf YG, Bocher M, Basevitch A, Rudis E, et al. Preoperative Thallium Scanning, Selective Coronary Revascularization, and Long-Term Survival After Major Vascular Surgery. Circulation. 2003;108:177-83. doi:10.1161/01.CIR.0000080292.11186.FB.

8. Yin S, Chou F-F, Wu S-C, Chi S-Y. Applying preoperative dipyridamole thallium-201 scintigraphy for preventing cardiac mortality and complications for patients with secondary hyperparathyroidism undergoing parathyroidectomy. Asian J Surg. 2017. doi:10.1016/j.asjsur.2017.03.004.

9. Shiga $T$, Wajima $Z$, Ohe $Y$. Is operative delay associated with increased mortality of hip fracture patients? Systematic review, meta-analysis, and meta-regression. Can J Anesth Can d'anesthésie. 2008;55:146-54. doi:10.1007/BF03016088.

10. Cluett J, Caplan J, Yu W. Preoperative cardiac evaluation of patients with acute hip fracture. Am J Orthop (Belle Mead NJ). 2008;37:32-6. http://www.ncbi.nlm.nih.gov/pubmed/18309382. Accessed 19 Mar 2017. 
11. Marcantonio A, Steen B, Kain M, Bramlett KJ, Tilzey JF, lorio R. The Clinical and Economic Impact of Preoperative Transthoracic Echocardiography in Elderly Patients with Hip Fractures. Bull Hosp Jt Dis. 2015;73:239-42. https://www.ncbi.nlm.nih.gov/pubmed/26630466.

12. Simunovic N, Devereaux PJ, Bhandari M. Surgery for hip fractures: Does surgical delay affect outcomes? Indian J Orthop. 2011;45:27-32. doi:10.4103/0019-5413.73660.

13. Middleton RG, Uzoigwe CE, Young PS, Smith R, Gosal HS, Holt G. Peri-operative mortality after hemiarthroplasty for fracture of the hip. Bone Joint J. 2014;96-B:1185-91. doi:10.1302/0301620X.96B9.33935.

14. Rodriguez-Fernandez P, Adarraga-Cansino D, Carpintero P. Effects of Delayed Hip Fracture Surgery on Mortality and Morbidity in Elderly Patients. Clin Orthop Relat Res. 2011;469:3218-21. doi:10.1007/s11999-010-1756-z.

15. Sheehan KJ, Sobolev B, Guy P, Kuramoto L, Morin SN, Sutherland JM, et al. In-hospital mortality after hip fracture by treatment setting. CMAJ. 2016;188:1219-25. doi:10.1503/cmaj.160522.

16. Ricci WM, Rocca GJDella, Combs C, Borrelli J. The medical and economic impact of preoperative cardiac testing in elderly patients with hip fractures. Injury. 2007;38 SUPPL. 3:49-52.

17. Sawhney C, Trikha V, Janani S, Bajwa SS, Sharma V, Khanna M. Impact of preoperative echocardiography on perioperative management in geriatric hip trauma: A retrospective observational study. Int J Appl Basic Med Res. 2017;7:104. doi:10.4103/2229-516X.205816.

18. Mutlu H, Bilgili F, Mutlu S, Karaman O, Cakal B, Ozkaya U. The effects of preoperative non-invasive cardiac tests on delay to surgery and subsequent mortality in elderly patients with hip fracture. $J$ Back Musculoskelet Rehabil. 2016;29:49-54. doi:10.3233/BMR-150595.

19. O'hEireamhoin S, Beyer T, Ahmed M, Mulhall KJ. The Role of Preoperative Cardiac Investigation in Emergency Hip Surgery. J Trauma Inj Infect Crit Care. 2011;71:1345-7. doi:10.1097/TA.0b013e318224cfa3.

20. Vigoda MM, Sweitzer B, Miljkovic N, Arheart KL, Messinger S, Candiotti K, et al. 2007 American College of Cardiology/American Heart Association (ACC/AHA) guidelines on perioperative cardiac evaluation are usually incorrectly applied by anesthesiology residents evaluating simulated patients. Anesth Analg. 2011;112:940-9.

21. Fathala A. Myocardial Perfusion Scintigraphy: Techniques, Interpretation, Indications and Reporting. Ann Saudi Med. 2011;31:625-34.

22. Heinle I, Siraj QH. Artefacts and pitfalls in myocardial perfusion imaging. Integr Cardiol Nucl Med Physicians A Guid to Nucl Med Physicians. 2009;:325-42.

23. Aziz EF, Javed F, Alviar CL, Herzog E. Triple vessel coronary artery disease presenting as a markedly positive stress electrocardiographic test and a negative SPECT-TL scintigram: a case of balanced Ischemia. Heart Int. 2011;6:81-5.

24. Hwang J, Kim E-K, Yang J-H, Chang S-A, Song YBin, Hahn J-Y, et al. Assessment of perioperative cardiac risk of patients undergoing noncardiac surgery using coronary computed tomographic angiography. Circ Cardiovasc Imaging. 2015;8:e002582. doi:10.1161/CIRCIMAGING.114.002582. 


\section{Tables}

\section{Table 1}

Baseline demographic data, surgical factors, and length of stay in unmatched and matched patients who did and did not undergo a dipyridamole-thallium scan.

\begin{tabular}{|c|c|c|c|c|c|}
\hline \multicolumn{2}{|c|}{ Thallium scan group ( $n=113)$} & \multicolumn{4}{|c|}{ Non-thallium scan group } \\
\hline & & \multirow{2}{*}{$\begin{array}{l}\begin{array}{l}\text { Unmatched cohort } \\
(n=731)\end{array} \\
74.7 \pm 11.3\end{array}$} & \multirow{2}{*}{$\begin{array}{l}p \text {-value } \\
<0.001\end{array}$} & \multirow{2}{*}{$\begin{array}{c}\begin{array}{c}\text { Matched cohort } \\
(n=339)\end{array} \\
79.9 \pm 8.3\end{array}$} & $p$-value \\
\hline Age, mean \pm SD, y & $79.8 \pm 8.3$ & & & & 0.901 \\
\hline Sex n (\%) & & & 0.590 & & 0.908 \\
\hline Male & $37(32.7)$ & $221(30.2)$ & & $109(32.2)$ & \\
\hline Female & $76(67.3)$ & $510(69.8)$ & & $230(67.8)$ & \\
\hline Body mass index, mean $\pm \mathrm{SD}, \mathrm{kg} / \mathrm{m}^{2}$ & $22.6 \pm 3.5$ & $22.7 \pm 4.1$ & 0.774 & $22.5 \pm 3.9$ & 0.980 \\
\hline ASA grade n (\%) & & & $<0.001$ & & 0.010 \\
\hline 1 & $0(0.0)$ & $11(1.5)$ & & $2(0.6)$ & \\
\hline 2 & $5(4.4)$ & $185(25.3)$ & & $57(16.8)$ & \\
\hline 3 & $106(93.8)$ & $523(71.5)$ & & $270(79.6)$ & \\
\hline 4 & $1(0.9)$ & $10(1.4)$ & & $8(2.4)$ & \\
\hline Duration to surgery, mean $\pm S D, d$ & $7.0 \pm 12.4$ & $2.4 \pm 3.3$ & $<0.001$ & $3.7 \pm 4.2$ & $<0.001$ \\
\hline Duration to surgery $>2$ days, $\mathrm{n}(\%)$ & $110(97.3)$ & $352(48.2)$ & $<0.001$ & 127 (37.5) & $<0.001$ \\
\hline \multicolumn{6}{|l|}{ Preoperative comorbidities, n (\%) } \\
\hline Ischemic heart disease & $25(22.1)$ & $56(7.7)$ & $<0.001$ & 47 (13.9) & 0.038 \\
\hline $\mathrm{CHF}$ & $6(5.3)$ & $27(3.7)$ & 0.431 & $20(5.9)$ & 0.816 \\
\hline Valvular heart disease & $11(9.7)$ & $18(2.5)$ & 0.001 & $17(5.0)$ & 0.071 \\
\hline CVA & $11(9.7)$ & $76(10.4)$ & 0.829 & $34(10.0)$ & 0.928 \\
\hline Diabetes & $44(38.9)$ & $216(29.5)$ & 0.044 & $123(36.3)$ & 0.613 \\
\hline COPD & $13(11.5)$ & $53(7.3)$ & 0.117 & $31(9.1)$ & 0.464 \\
\hline RA & $1(0.9)$ & $14(1.9)$ & 0.440 & $3(0.9)$ & 1.000 \\
\hline Liver disease & $2(1.8)$ & $37(5.1)$ & 0.121 & $7(2.1)$ & 1.000 \\
\hline Renal disease & $14(12.4)$ & $59(8.1)$ & 0.129 & 37 (10.9) & 0.668 \\
\hline Cancer & $4(3.5)$ & $48(6.6)$ & 0.213 & $12(3.5)$ & 1.000 \\
\hline Anesthesia type, n (\%) & & & 0.071 & & 0.597 \\
\hline General & $94(83.2)$ & $651(89.1)$ & & $289(85.3)$ & \\
\hline Regional & $19(16.8)$ & $80(10.9)$ & & $50(14.7)$ & \\
\hline Cemented stem, n (\%) & $65(57.5)$ & $297(40.6)$ & $<0.001$ & $199(58.7)$ & 0.826 \\
\hline Time in surgical theatre, mean $\pm \mathrm{SD}$, & $125.9 \pm 36.3$ & $132.3 \pm 44.5$ & 0.142 & $125.5 \pm 33.8$ & 0.930 \\
\hline
\end{tabular}

ASA, American Society of Anesthesiologists; CHF, congestive heart failure; CVA, cerebrovascular accident; COPD, chronic obstructive pulmonary disease; RA, rheumatoid arthritis; SD, standard deviation. 


\section{Table 2}

Univariate and multivariate logistic regression models evaluating the associations of dipyridamole-thallium scan with 90-day cardiac complications and mortality.

\begin{tabular}{|c|c|c|c|c|c|c|}
\hline \multirow[t]{2}{*}{ Outcome } & \multicolumn{4}{|c|}{ Unmatched cohort } & \multicolumn{2}{|l|}{ Matched cohort } \\
\hline & $\begin{array}{l}\text { Unadjusted OR } \\
(95 \% \mathrm{CI})\end{array}$ & $p$-value & $\begin{array}{l}\text { Adjusted OR* } \\
(95 \% \mathrm{CI})\end{array}$ & $p$-value & $\begin{array}{l}\text { Adjusted OR† } \\
(95 \% \mathrm{CI})\end{array}$ & $p$-value \\
\hline Overall & $1.86(1.11-3.13)$ & 0.019 & $1.44(0.71-2.91)$ & 0.309 & $1.32(0.75-2.33)$ & 0.332 \\
\hline \multicolumn{7}{|l|}{ Cardiac complications } \\
\hline Unstable angina & $6.52(0.41-104)$ & 0.186 & $4.26(0.12-154)$ & 0.429 & $3.0 \quad(0.19-48)$ & 0.437 \\
\hline Acute myocardial infarction & $3.28(0.59-18.1)$ & 0.174 & $2.83(0.38-21.2)$ & 0.310 & $3.0 \quad(0.42-21.3)$ & 0.272 \\
\hline Mortality & $0.92(0.11-7.58)$ & 0.941 & $(0.23-424.8)$ & 0.235 & $0.75(0.08-6.71)$ & 0.797 \\
\hline
\end{tabular}

*Adjusted for age, sex, body mass index, American Society of Anesthesiologists (ASA) grade, preoperative comorbidities (ischemic heart disease, congestive heart failure, valvular heart disease, cerebrovascular accident, diabetes, chronic obstructive pulmonary disease, rheumatoid arthritis, liver disease, renal disease, and cancer history), and surgical variables (anesthesia and cemented stem, operative duration, and transfusion), and duration to surgery.

†Adjusted for ASA grade and ischemic heart disease.

OR, odds ratio; CI, confidence interval.

\section{Table 3}

Results of multivariate logistic regression regarding 90-day cardiac complications.

\begin{tabular}{lcccc}
\hline & \multicolumn{1}{c}{ Beta coefficient Standard error } & OR & $95 \%$ CI & $p$-value \\
\hline ASA grade 3 & 1.16 & 0.41 & $3.19(1.44$ to 7.08$)$ & 0.004 \\
\hline Cardiac comorbidities & 1.72 & 0.26 & $5.56(3.35$ to 9.25$)$ & $<0.001$ \\
\hline
\end{tabular}

Cardiac comorbidities included ischemic heart disease, congestive heart failure, and valvular heart disease. OR, odds ratio; CI, confidence interval; ASA, American Society of Anesthesiologists.

\section{Figures}




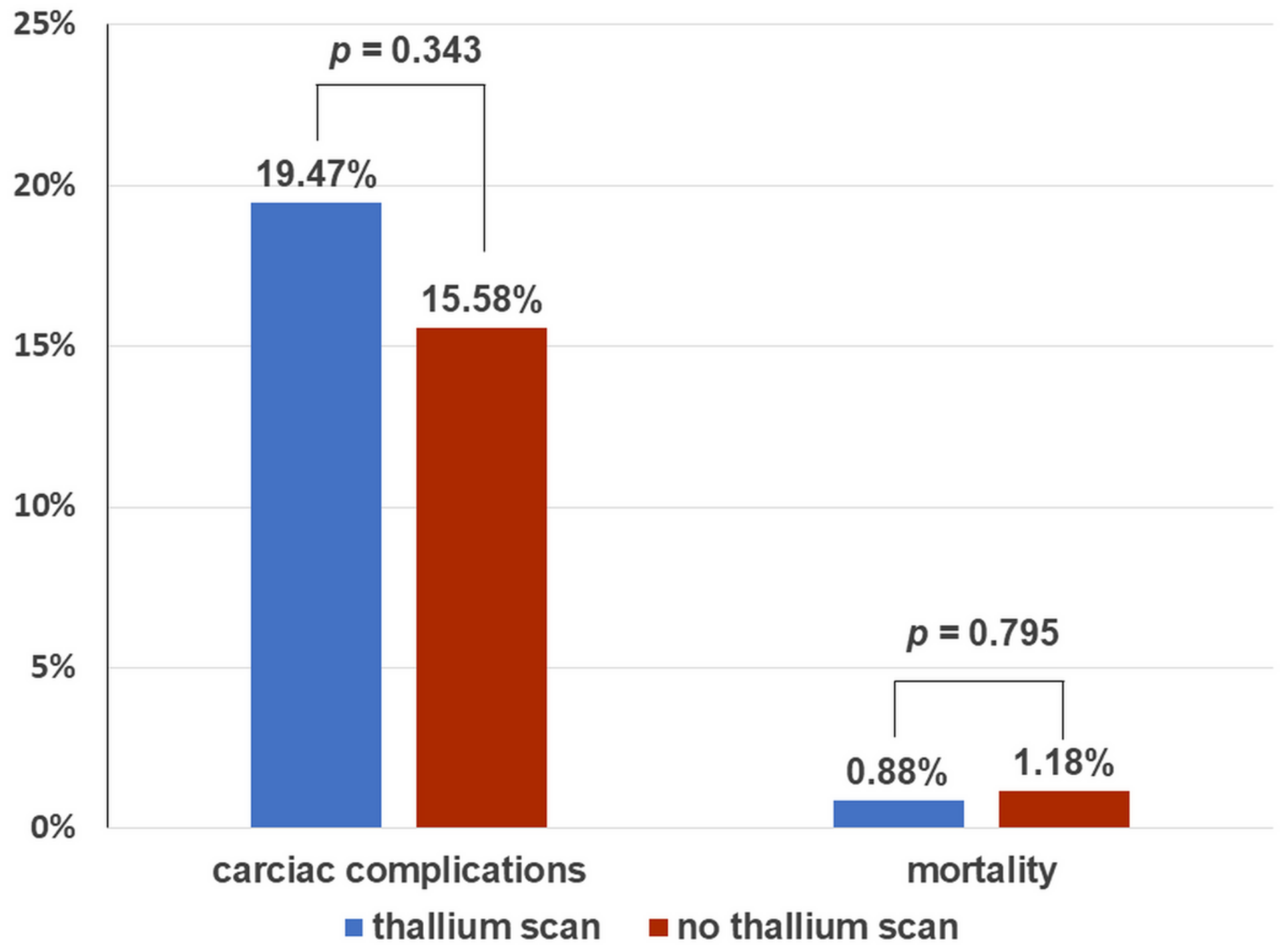

Figure 1

90-day cardiac complications and mortality in patients with and without a dipyridamole-thallium scan after propensity score-matching. 


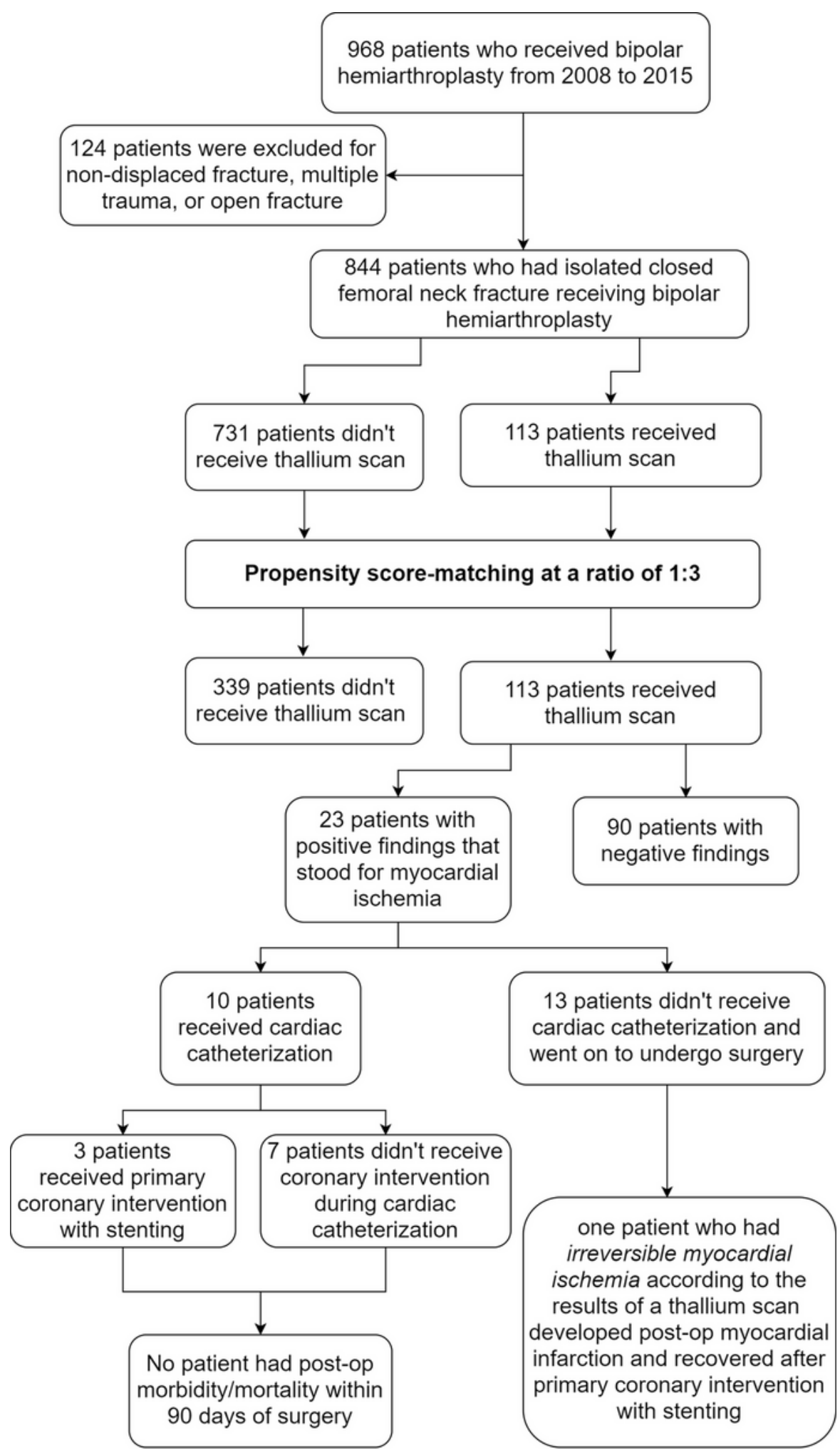

\section{Figure 2}

Flowchart showing the selection process of patients and the further decision-making in the two groups, categorized according to performance of a dipyridamole-thallium scan. 